\title{
Designing a Smart Monitoring and Alert System for Malaria Patients Based on IoT in Burundi
}

\author{
https://doi.org/10.3991/ijoe.v17i03.20369 \\ Igor Didier Sabukunze ${ }^{\bowtie}$, Suyoto \\ Universitas Atma Jaya Yogyakarta, Yogyakarta, Indonesia \\ sigordidier2015@gmail.com
}

\begin{abstract}
Malaria is an infectious disease that has a devastating effect on humans but is contagious and preventable. There were 228 million malaria cases globally in 2018, with $93 \%$ of patients and 94\% dying showing up in Africa within Burundi, possessing a massive growth (51\%). This study proposes an IoT design for smart monitoring and alert systems for malaria patients in $\mathrm{Bu}$ rundi. After being tested positive while they are home drinking tablets prescribed by the doctor, the patients will use a body temperature sensor. When the situation worsens, the patient shall be hospitalized and use the water level sensor to monitor the serum level, which contains quinine. Body temperature and water level sensor will be connected to Arduino Microcontroller, and all information will be gathered and sent to the IoT server through the internet. The system design is authentic and adequate in reducing mortality in Burundi. Besides, the system is equipped to set up a caution message to the medical staff in case of the patient's extreme conditions. It can be concluded that a Smart monitoring and alert system is essential for monitoring malaria patients and can alert in case of a severe condition.
\end{abstract}

Keywords - IoT, malaria, alert system, monitoring

\section{Introduction}

Malaria is an infectious disease that has a significant impact on people, but it does spread, although it is protected. The malaria epidemic in Burundi continues, with crisis issues persisting throughout the country. As the 2019 Universe Malaria article reports, there were 228 million malaria cases worldwide in 2018, with $93 \%$ of patients and $94 \%$ of deaths occurring in Africa [1]. Some countries observed growth in types in the middle of 2015 and 2018, with Burundi possessing a massive expansion (51\%) [1]. But a recent technology, the so-called Internet of Things (IoT), has increased the internet's ability to take over computer equipment. An IoT design, followed by an IoT application, should be built to eradicate malaria because it is essential to monitor the patient using technology to reduce Burundi's death rate.

Mosquitoes are associated with local characteristics environment (e.g., standing water or irrigation system), human behavior (water communication design), and socioeconomic conditions (ability to maintain a clean environment) [2]. Gaining a better 
understanding of malaria causes, mode of transmission, signs and symptoms, malaria complications, and malaria prevention leads to malaria prevention methods [3]. Malaria not only negatively affects society's growing economy but the result of poverty.

The medical zone is not neglected in IoT technology's general use because IoT technology is rapidly rising [4] and becoming popular in this regard. Patient health observing is defined as continuous monitoring of clinical studies conducted to replace with a set of well-established clinical practice guidelines, standard operating procedures, and needs. This health has become a much-needed program amount of time to generate, analyze, and disseminate information. IoT can improve health and wellbeing [5] through better efficiency and improved care in health facilities, through the ability to allow for the use of a wide range of remote sensing tools, allowing people to monitor their health daily [6] in Burundi.

The proposed design aims to control the serum of malaria patients' temperature and status in a room where an automatic body temperature sensor and water detector/ sensor will be connected to Arduino to check the serum's quality in a Burundi hospital. This automated system can be monitored from a mobile application by a doctor or nurse.

\section{Related Works}

Most of the styles are intuitive. However, we invite you to read carefully the brief description below.

According to A. Yogaraj, a system has been designed [7] to reduce rural hardships people[8]. This system was significantly reducing human effort by connecting remote locations [9] to people. The sensors play an essential role in this diminished health care facility. A model LM-35 of a temperature sensor has been utilized to measure the temperature of a patient. The heart rate of the human body is calculated by the heartbeat [10]. Using an ECG sensor [11] module is connected to check the heartbeat and blood flowing inside. All those sensors together are associated with the Arduino.

Deep Learning methods such as image classification, video identification, and clinical imaging continue to be used to detect malaria. Convolutional neural networks $(\mathrm{CNN})$, a deep neural network, are often used to evaluate studies in remote sensing computers [12]. According to S. K. Sood and I. Mahajan, a Smart IoT system based on the Neutral Point Controller was used to reduce internal and external portfolios [13].

The physical space and cyberspace system that differentiate, diagnose, and prevent MBDs (Mosquito-Borne Diseases) contain two areas. A corporal room oversees the collection of specific environmental information and health data also the distribution of all-inclusive work to all stakeholders, including users, health services, and health professionals. In contrast, cyberspace contains two parts, such as the fog part and cloud compounds. The received information is provided in the cloud section for immediate actions also identifies potential operators. After detecting the MBD, the fog parts quickly make notifications on the operator's handphone to issue alerts at a time [14]. 
The architecture of an intelligent system [15] for early detection and prevention of mosquito [16]-borne diseases has been made. The F-HMRAS(Fog-based Health Monitoring and Risk Assessment System) was for distinguishing and detect exact mosquito-borne diseases and cause alerts or warning in the event of an emergency to prevent infection [17].

A smart program to make radiation-appropriate measures has been designed and implemented. The IoT was also used through a communication gamma meter and a cheap U.V. radiation machine [18]. Biosensors that are simple, accessible, and economically applicable outside the laboratory by inexperienced people have been developed to detect early malaria status [19]. In the study of M. Campion et al. [20], an example predicts the number of traps and the evolution of a convenient netcommunication holding collaborative internet pages that permit operators to see North Dakota mosquitoes and climate plans WNV rate records are shown.

Neural networks and fuzzy systems were recommended to be integrated into A.I.'s health control safety to simulate a smart device test to select the priority based on the sensor nodes' health collection factors [21]. An intelligent healthcare monitoring system (SHMS) uses the WBSN derived from medical sensors to gather inside and outside physiological information. This system had two fundamental origins of information, the first origin was the wireless body sensor Network (WBSN), and another data origin was EMRs. EMRs furnish patient monitoring reports, medical history, etc. [22].

A conception of an intelligent health support system for patient surveillance in quarantines, application of IoT based smart home healthcare for decreasing needless trouble on hospitals caused by infectious diseases, and removal and explanation of patient health information from wearable [4], incorporated, and portable sensors for medical diagnostic and treatment made by Taiwo and Absalom E. Ezugwu [23]. Recently, S. Abbas et al. [24] presented a controlling system using intelligent sensing devices attached to more than 8000 trees in Hong Kong's rural-urban areas landscape. And Basker and Anand [25] gave a smart monitoring system for asthma patients.

\section{Proposed System}

The manifestation of malaria is like the flu, high temperature, coldness, sweating, migraine, nausea, vomiting, and many more. Without early diagnosis and treatment, malaria can progress rapidly to severe illness and death.

The patient with such symptoms should be rushed to the hospital for a medical check-up. After being tested, the patient should return to take the results a few hours ago, along with a medical record of medications he/she needs to take according to his illness. Often in Burundi, malaria patients undergo treatment by drinking quinine in their early stages or other medication, but mostly it is quinine.

Figure 1 shows the proposed Smart Monitoring System, and Figure 2 shows its Flowchart. A patient will be given medication prescribed by medical personnel (Doctor/ nurse) to take at home. A body temperature sensor connected to him to generate data from him and be displayed to the smartphone operates by the patient guardian. 
The information will transit from this smartphone through the internet until the IoT server. The database manager will access the data to see any change in the patientrelated to his temperature. If the temperature exceeds or goes down to the average temperature, vomiting many times, and all those signs make the patient wick. A patient guardian who is with the patient will contact the medical team through the mobile application. The application lets them know about the situation of the patient. The medical team will also be aware because the medical team's application alert notification will be given in the remote device managed by a database manager. The database manager will inform the doctor/nurse who is in charge of his smartphone to decide if an ambulance can pick up the patient from his home or reach the hospital with his patient guardian's help using another way of transportation.

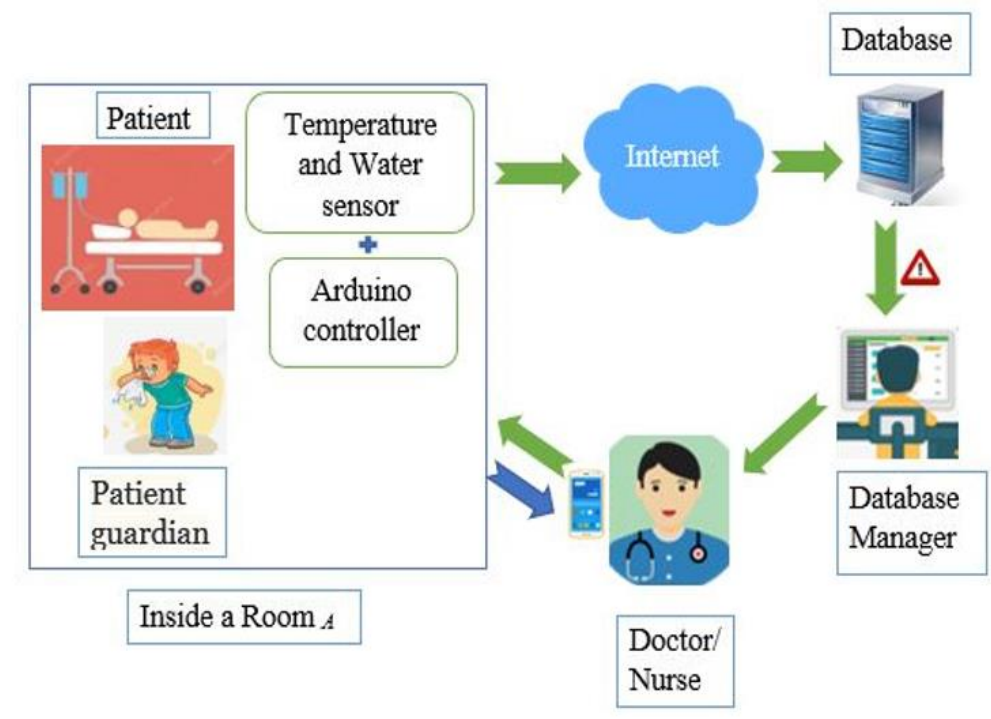

Fig. 1. Proposed Smart Monitoring System

In this part, the author will describe how the design will work.

This design started to be functional when the patient is already in the hospital for treatment because of his status; instead of becoming well, it is worsened while drinking tablets. By there, he should be treated in the hospital undergo a serum that contains quinine. Data transmission will be done via Bluetooth technology and the internet. And IoT devices that will be used for supporting the information of data are the body temperature sensor, water level sensor, and Arduino controller.

Room A indicates rooms available for malaria patients in a Hospital in Burundi.

The minimum timing for the serum to be changed is 8 hours, but it can take more than 8 hours to run out for other reasons. Therefore, to know whether the serum has finished, a water sensor will generate information on the serum's status. A body temperature sensor will also be used to see if the patient's temperature increases or de- 
creases. All those two sensors will be connected to an Arduino Controller, which will generate information from those sensors and send them to the patient's guardian's smartphone through Bluetooth. The data will transit until an IoT server via the internet, where a database manager can have access. A notification message will be displayed on the database manager's screen in a higher or lower temperature or a finished serum. And this one will inform the doctor or the nurse in charge so that they can intervene immediately before the patient's guardian alerts them. However, physicians and patients/patients' guardians can see individual information conveyed to the IoT Server using the mobile application or web. The Emergency hospital units' users can access the mobile application through their Access ID and secret code.

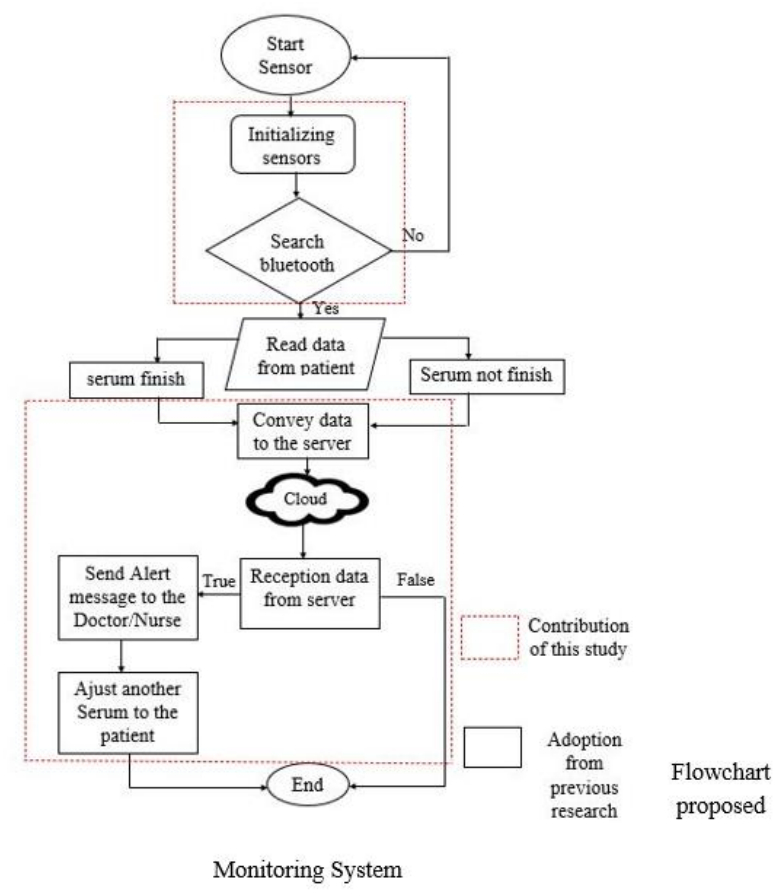

Fig. 2. Flowchart of the proposed Smart Monitoring System

\section{$4 \quad$ Result and Discussion}

In Figure 3, a detailed overview of the patient's different sensors has been presented in Figure 2 to display the smartphone's data until reaching the hospital monitor. The body temperature sensor will be worn on his body, and the Water level Sensor should be placed into the patient's serum to detect serum level. Both two sensors will collect information and send it to the smartphone through an Arduino controller on which they are connected. 
This system has implemented different sensors to monitor the patient's health in a hospital ward. These sensors are adaptable to the Arduino controller Uno.

The cause of adopting an Arduino-based smart system is just because it is extensively accessible, cheaper, and simple to apply.

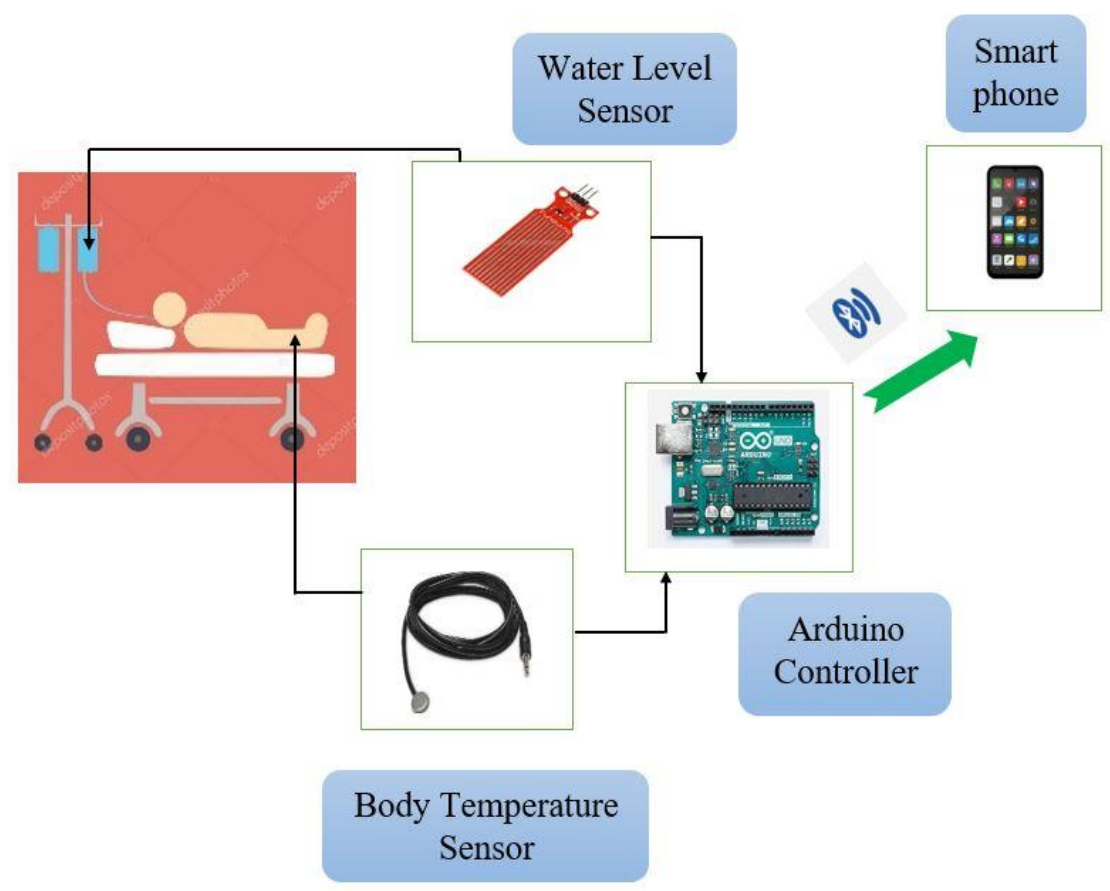

Fig. 3. Sensor placement design used by a patient

Body temperature sensor: (YSI 400AC): In this study, YSI 400 Series Reusable Temperature Probes has been chosen to be applied because probes have a much lower cost in use than any cheap product or automation. Usable skin surface probes have exceptional temperature feedback. The Temperature probe has a head length of 2 meters and is designed to operate over a range of 0 to $60^{\circ} \mathrm{C}$. The information obtained from the patient will be conveyed directly to a patient monitor via a wire of the body temperature sensor, which is connected to Arduino Uno, then will be transferred to an IoT server. This information will be displayed on the doctor's remote monitoring. The acceptable body temperature for the human body is $37^{\circ}$ Celsius.

Water level sensor pinout (DC3-5V): Water fluid sensor is used to track and determine the position of water in free space. The water level sensor is excellent, simple to operate, and has only 3 pin to associate. An analog output S must be linked to one of the analog inputs, which is on Arduino. This sensor will be sunk in a serum that contains quinine and connected to a patient. This sensor directly transfers information 
of serum status through cables that link the sensor to Arduino. Then Arduino will convey information directly into the patient's smartphone before the data reaches into IoT server through the cloud. From there, the Database manager can get access to the information quickly.

Arduino UNO microcontroller: Arduino UNO is used in our system because it is the microcontroller's legal basis. The Arduino Uno is a basic microcontroller based on the ATmega328. It possesses 14 numerical input/output pins (including 6 PWM outputs), six analog inputs, $16 \mathrm{MHz}$ ceramic resonators, a USB connection, a power outlet, and much more. This prototype of Arduino can gather analog and numerical information [26]. Arduino is used to collecting data from sensors; depend on the data, the microcontroller conveys the information to the equivalent real command devices and alert system [27].

Database: The database server is responsible for running a network database application and managing database files. A database is needed in a hospital to manage patient records to store data for a long time.

Database manager: A database manager is a person who is responsible for running the whole system of a business organization. A hospital database manager must monitor the patient's data to let them know the doctor/nurse in patients' severe condition using IoT devices. If a patient is in critical condition, the database manager will check in the doctor/nurse's system during that time in that service and send the message to him so that the patient s' life can be saved.

Design of alert notification: In Figures 4 and 5, two sensors have been used to show how notifications will be displayed on the monitor/smartphone operated by the patient guardian and on the medical team's side in case of emergency.

In Figure 4, two notifications received indicate that patient 01 and patient 02 their temperature has increased, while the second notification shows that patient 03 and patient 04 their temperature has decreased. Usually, the body temperature rises during exercise and varies with the environment's extreme temperatures because the temperature regulation mechanisms are not perfect. The acceptable temperature for the human body is 36.5 to $37.5^{\circ} \mathrm{C}$.

In fever, the central body temperature rises at high temperatures under the brain that regulates body temperature. In hyperthermia, the body temperature rises without the influence of the central heating system.

In the cold, body temperature can go under what is needed for normal bodily functions and metabolism. The condition is caused by additional risk to cold weather or water in humans but can be intentionally triggered as a medical treatment. 


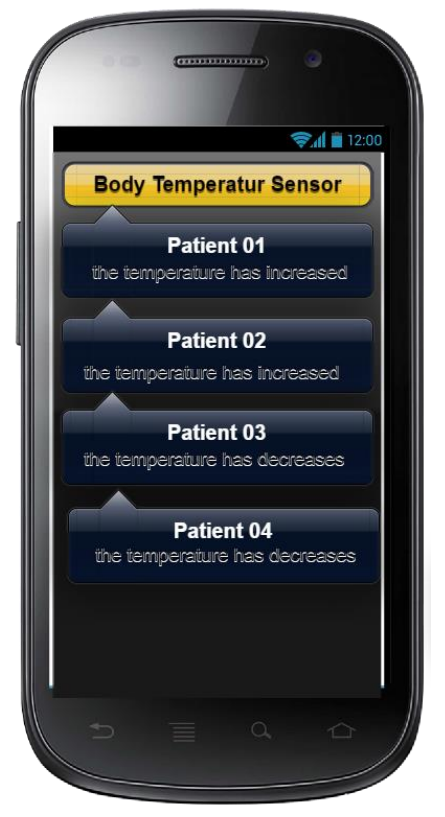

Fig. 4. Notification from the Body temperature sensor

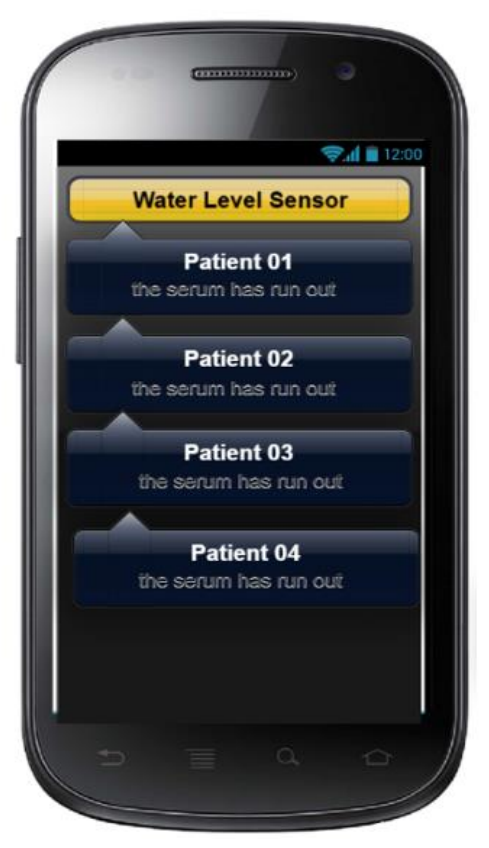

Fig. 5. Notification from the Water level sensor 
In Figure 5, the notification displayed shows that serum injected into the patients has run out. When information such as this is displayed on a doctor or nurse monitor, they have to rush to renew another to avoid complications to the patient, such as blood starting going up and getting into the patient's finished serum.

\section{Conclusion}

This suggested research is to monitor malaria patients located in Room A, in a hospital in Burundi. This patient starts to be observed after being tested positive for malaria. The patient will use a body temperature sensor to generate information from the patient and send it to the IoT server. Patients will be hospitalized; instead of getting well after a medical prescription, vomiting and other symptoms continue to show up. Patients will use two sensors while being in the hospital, which are body temperature sensors for monitoring the temperature and water level sensor to generate serum injected in the patient. This system can generate an alert notification in case of a medical team when the patient requires medical intervention when the serum is run out or in case of increasing or decreasing temperature.

Before the system was created, the medical team used to be called by the patient's guard in case of an emergency, namely when the serum ran out or the temperature rose or fell. But from now on, everything will be monitored from a distance.

For the system's success, it can be concluded that smart monitoring and emergency alert systems are essential for monitoring malaria patients and can alert in case of extreme patient conditions that require medical intervention.

\section{Acknowledgement}

The authors would like to thank the Universitas Atma Jaya Yogyakarta for supporting the research. Including anonymous reviewers for their endeavor to make this research excellent.

\section{$7 \quad$ References}

[1] Global Malaria Programme: WHO Global, World malaria report 2019. 2019.

[2] WHO, "Achieving good health," Focus (Madison)., pp. 7-18.

[3] A. Tamirat and M. Geremew, "Knowledge, Attitude and Practice about Malaria in Maji District, Bench Maji Zone, Southwest Ethiopia," J. Trop. Dis., vol. 4, no. 3, 2016, https://doi.org/10.4172/2329-891x.1000217.

[4] T. T. Sahidi, A. Basuki, and H. Tolle, "MIOT framework, general purpose internet of things gateway using smartphone," Int. J. Online Eng., vol. 14, no. 2, pp. 6-23, 2018, https://doi.org/10.3991/ijoe.v14i02.7326

[5] F. Sanfilippo and C. Pacchierotti, "A wearable haptic system for the health monitoring of elderly people in smart cities," Int. J. Online Eng., vol. 14, no. 8, pp. 52-66, 2018, https://doi.org/10.3991/ijoe.v14i08.8571. 
[6] P. Biggs, J. Garrity, and C. LaSalle, "Harnessing-IoT-Global-Development.pdf," p. 61, 2015.

[7] A. Yogaraj, M. R. Ezilarasan, R. V Anuroop, C. S. Sivanthiram, and S. K. Thakur, "IOT based smart healthcare monitoring system for rural/isolated areas," Int. J. Pure Appl. Math., vol. 114, no. 12 Special Issue, pp. 679-687, 2017, [Online]. Available: https:// www.scopus.com/inward/record.uri?eid=2-s2.0-85034024457\&partnerID=40\&md5=98ec d875b3ff83f35bd16b958bd29833.

[8] FAO, "Strategic work of FAO to," 2017, [Online]. Available: www.fao.org/european union/eu-projects/first/en/.

[9] Y. Li, S. Grimaldi, V. R. N. Pauwels, and J. P. Walker, "Hydrologic model calibration using remotely sensed soil moisture and discharge measurements: The impact on predictions at gauged and ungauged locations," J. Hydrol., vol. 557, pp. 897-909, 2018, https://doi.org/10.1016/j.jhydrol.2018.01.013.

[10] M. A. Mujawar, H. Gohel, S. K. Bhardwaj, S. Srinivasan, N. Hickman, and A. Kaushik, "Nano-enabled biosensing systems for intelligent healthcare: towards COVID-19 management," Mater. Today Chem., vol. 17, p. 100306, 2020, https://doi.org/10.1016/ j.mtchem.2020.100306

[11] A. Nadeem, A. Mehmood, and K. Rizwan, "A dataset build using wearable inertial measurement and ECG sensors for activity recognition, fall detection and basic heart anomaly detection system," Data Br., vol. 27, p. 104717, 2019, https://doi.org/10.1016/j.dib.2019.10 $\underline{4717}$

[12] M. Masud et al., "Leveraging Deep Learning Techniques for Malaria Parasite Detection Using Mobile Application," Wirel. Commun. Mob. Comput., vol. 2020, 2020, doi: $10.1155 / 2020 / 8895429$.

[13] S. Krishnan, P. Umasankar, and P. Mohana, "A smart FPGA based design and implementation of grid connected direct matrix converter with IoT communication," Microprocess. Microsyst., vol. 76, p. 103107, 2020, https://doi.org/10.1016/j.micpro.2020.103107

[14] S. K. Sood and I. Mahajan, "Fog-cloud based cyber-physical system for distinguishing, detecting and preventing mosquito borne diseases," Futur. Gener. Comput. Syst., vol. 88, pp. 764-775, 2018, https://doi.org/10.1016/j.future.2018.01.008

[15] M. Abdel-Basset, R. Mohamed, M. Elhoseny, and V. Chang, "Evaluation framework for smart disaster response systems in uncertainty environment," Mech. Syst. Signal Process., vol. 145, p. 106941, 2020, https://doi.org/10.1016/j.ymssp.2020.106941

[16] Z. T. Salim, U. Hashim, M. K. M. Arshad, M. A. Fakhri, and E. T. Salim, "Frequencybased detection of female Aedes mosquito using surface acoustic wave technology: Early prevention of dengue fever," Microelectron. Eng., vol. 179, pp. 83-90, 2017, https://doi. org/10.1016/j.mee.2017.04.016

[17] V. Vijayakumar, D. Malathi, V. Subramaniyaswamy, P. Saravanan, and R. Logesh, "Fog computing-based intelligent healthcare system for the detection and prevention of mosquito-borne diseases," Comput. Human Behav., vol. 100, pp. 275-285, 2019, https://doi.org/10.1016/j.chb.2018.12.009

[18] R. Baena-Navarro, F. Torres-Hoyos, C. Uc-Rios, and R. F. Colmenares-Quintero, "Design and assembly of an IoT-based device to determine the absorbed dose of gamma and U.V. radiation," Appl. Radiat. Isot., vol. 166, p. 109359, 2020, https://doi.org/10.1016/j.apradis $\underline{0.2020 .109359 \text {. }}$

[19] G. Dutta, "Electrochemical biosensors for rapid detection of malaria," Mater. Sci. Energy Technol., vol. 3, pp. 150-158, 2020, doi: 10.1016/j.mset.2019.10.003. 
[20] M. Campion et al., "Predicting West Nile Virus (WNV) occurrences in North Dakota using data mining techniques," FTC 2016 - Proc. Futur. Technol. Conf., no. December, pp. 310317, 2017, https://doi.org/10.1109/ftc.2016.7821628

[21] H. A. El Zouka and M. M. Hosni, "Secure IoT communications for smart healthcare monitoring system," Internet of Things, no. February 12, p. 100036, 2019, https://doi.org/ 10.1016/j.iot.2019.01.003

[22] F. Ali et al., "A smart healthcare monitoring system for heart disease prediction based on ensemble deep learning and feature fusion," Inf. Fusion, vol. 63, pp. 208-222, 2020, https ://doi.org/10.1016/j.inffus.2020.06.008.

[23] O. Taiwo and A. E. Ezugwu, "Smart healthcare support for remote patient monitoring during covid-19 quarantine," Informatics Med. Unlocked, vol. 20, p. 100428, 2020, https ://doi.org/10.1016/j.imu.2020.100428

[24] S. Abbas et al., "Tree tilt monitoring in rural and urban landscapes of Hong Kong using smart sensing technology," Trees, For. People, vol. 2, p. 100030, 2020, https://doi.org/10. 1016/j.tfp.2020.100030.

[25] N. S, H. S.K, I. Z. Basker, and D. J. Anand, "Smart Monitoring System For Asthma Patients," Int. J. Electron. Commun. Eng., vol. 7, no. 5, pp. 5-9, 2020, doi: 10.14445/23488549/ijece-v7i5p102.

[26] O. H. Yahya, H. T. S. Alrikabi, and I. A. Aljazaery, "Reducing the data rate in internet of things applications by using wireless sensor network," Int. J. online Biomed. Eng., vol. 16, no. 3, pp. 107-116, 2020, https://doi.org/10.3991/ijoe.v16i03.13021

[27] A. I. Sourav, N. D. Lynn, and Suyoto, "Smart monitoring system design for perishable food supply chain management based on IoT in Bangladesh," Int. J. Adv. Sci. Technol., vol. 29, no. 1, pp. 1069-1079, 2020.

\section{Authors}

Igor Didier Sabukunze is a student in the Informatics Department, Faculty of Industrial Technology, Universitas Atma Jaya Yogyakarta, Yogyakarta 55281, Indonesia. He undergraduate in Computer Science from Hope Africa University in Burundi. His interests are Mobile Application, Gamification, Computer Networking, Artificial Intelligence, and the Internet of Things. Email: sigordidier2015@gmail.com

Suyoto is a Professor in the Informatics Department at Universitas Atma Jaya Yogyakarta, Yogyakarta, 55281, Indonesia. He has more than twenty years of teaching experience. He received his Ph.D. in 2000 from the National University of Malaysia, Malaysia. His research interests are multimedia, computer graphics, visualization, Mobile Application, and Artificial Intelligence. Email: suyoto@staff.uajy.ac.id

Article submitted 2020-12-08. Resubmitted 2021-01-14. Final acceptance 2021-01-16. Final version published as submitted by the authors 